\title{
An outline of the use of supramolecular compounds in biology and medicine*
}

\author{
Anna Jagusiak $\bowtie$ \\ ${ }^{1}$ Chair of Medical Biochemistry, Faculty of Medicine, Jagiellonian University Medical College, Kraków, Poland
}

\begin{abstract}
Supramolecular chemistry opened up opportunities and prospects in biological studies. Numerous supramolecular systems are known which assume different shapes depending on the structure of the component molecules. Some of them are mixed systems. Ribbon-like supramolecular structures which are the main focus of this paper form complexes with proteins in a way which is a new type of interaction. Despite preserving the ribbonlike structure, it may also interact with proteins as a mixed system. In this way, it may be used as a carrier of drugs. In addition, hybrid systems created by a combination of ribbon-like supramolecular structures with carbon nanotubes were presented together with data from studies of these structures as drug-carrying systems. This paper presents an outline of the experimentally confirmed possible use of ribbon-like supramolecular systems.
\end{abstract}

Key words: supramolecular chemistry, host-guest recognition, self-delivery system, protein binding, Congo red

Received: 08 November, 2019; revised: 01 December, 2019; accepted: 04 December, 2019; available on-line: 28 December, 2019

$\bigotimes_{\text {e-mail: anna.jagusiak@uj.edu.pl }}$

*Acknowledgements of Financial Support:

The costs of the article published as a part of the 44th FEBS Congress Kraków 2019 - From molecules to living systems block are financed by the Ministry of Science and Higher Education of the Republic of Poland (Contract 805/P-DUN/2019).

This work was financially supported by the National Science Centre, Poland (grant \# 2016/21/D/NZ1/02763).

Abbreviations: $\mathrm{Ag}-\mathrm{Ab}$, antigen-antibody complex; $\mathrm{CR}$, Congo red; SRBC, Sheep Red Blood Cells; $L \lambda$, IgG light chain $\lambda ; T Y$, Titan yellow

\section{INTRODUCTION}

Supramolecular chemistry describes the phenomena of self-association of individual molecules to form large aggregates. Their formation is based on non-covalent bonds and hydrophobic interactions in particular. Supramolecular systems formed as a result of these interactions are composed of a large number of molecules and include mono- and polymoieties, micelles, vesicles, liposomes, liquid crystals and also inclusive complexes of host-guest type and small aggregates containing several molecules only as well (Atwood 2004, Xin et al., 2019). Supramolecular chemistry is used in many disciplines of biology and medicine. It represents structures which may be used as adsorbents of gases and many other poorly soluble substances. In such cases, they may play a role of carriers for drugs or active substances such as cosmetics for example (Kang et al., 2002; Chen et al., 2016; Webber et al., 2016; Yu et al., 2017; Xin et al., 2019). Of special interest appeared supramolecular structures of ribbon-like configuration. They are formed by sym- metric, planar, polyaromatic ring molecules as Congo red for example. The supramolecular structures of this kind penetrate and anchor within the protein body if it is unstable and hence susceptible to the penetration of large ligands (Roterman \& Konieczny, 2018). Instability which is correlated with the function of protein is tightly connected to the dye complexation with protein activity. Antibodies in the immune complex are an example of such proteins. They may be used in the immune-targeting system directed, among others, to tumors. The selectivity of the carriers can be achieved by using antibodies which bind to receptors overexpressed on tumor cells (Piekarska et al., 2004).

\section{SUPRAMOLECULAR SYSTEMS}

Interaction with proteins and nucleic acids is a hallmark of supramolecular systems. Calixarenes have been employed for protection, activation or inhibition of enzymes. These systems are also useful as anticoagulants, for specific binding to lectins and detection of the pathogenic prion protein. Formation of the double helix of DNA from complementary single polynucleotide strands is one of the better-known self-association processes in nature.

The complexes with metals which are sensitive to allosteric control lead to formation of new bioactive DNA ligands. These ligands allow for selective splitting the sequences. Cancer treatment with platinum derivatives is one of the more interesting applications of metal-bearing complexes. Those studies revealed how the addition of functional groups in such complexes could significantly increase their efficacy and be promising from a biological targeting perspective. New derivatives of platinum can help solve problems with its toxicity. Selectivity can be achieved by using ligands which bind to receptors hyperexpressed in tumors (Wells et al., 2007; Schneider, 2013).

Supramolecular structures are multi-molecular associates formed through non-covalent interactions, which contributes to their elasticity. The shape of the final

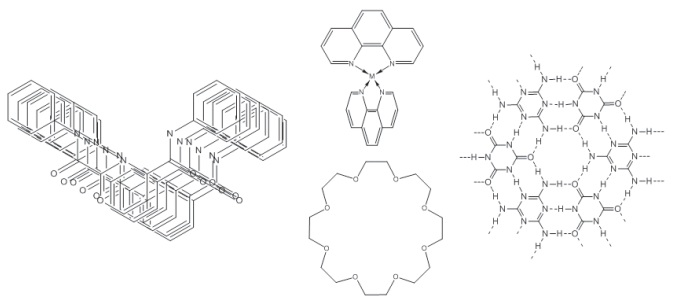

Figure 1. Examples of supramolecular systems consisting of individual molecules that form different angles during association resulting in bent, circular or spherical structures 
supramolecular structures depends on their component molecules. Single molecules that associate under various angles form bent, circular or spherical final structures. Unidirectionally aligned molecules create membranous or cross-linked forms (Fig. 1).

On the other hand, flat, elongated, symmetrical molecules form ribbon-like structures. The different ribbon-like systems have been described in many publications, but new applications and possible discoveries in this field are still in progress (Roterman et al., 2018). Below we present an overview of their possible applications in interaction with proteins, as potential drug carriers, with carbon nanotubes, and in imaging techniques.

\section{RIBBON-LIKE SUPRAMOLECULAR SYSTEMS: INTERACTION WITH PROTEINS}

Proteins usually perform their function by the formation of complexes with other proteins or reactions with low-molecular-weight organic or inorganic ligands. Complexation of ligands in biology is usually limited to a certain area termed the active site but the actual binding site is formed in contact with the ligand to match its structure. By folding, polypeptide chain forms a coherent structure stabilized at the so-called global minimum. Thus, the protein is a coherent structure. A local change in the structure within the active site induces alterations in the molecule outside of the active site. These structural changes are a sign of destabilization because after the ligand release the structure returns to the initial form. The presence of unstable areas in the protein facilitates the penetration of different chemical compounds while non-specific interactions of these compounds are weak and structure destabilization induced by ligand binding cannot be preserved after its release. An appropriate ligand which would be capable of preventing the protein return to its initial form should be large in order to ensure multi-point contact and concomitantly elastic to allow for structural changes of the ligand to match the surroundings. Ligands able to meet these requirements were found among supramolecular structures. Congo red (CR) is a typical representative of supramolecular com-

A)

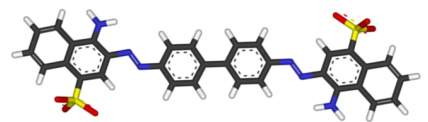

B)

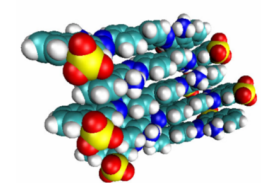

c)
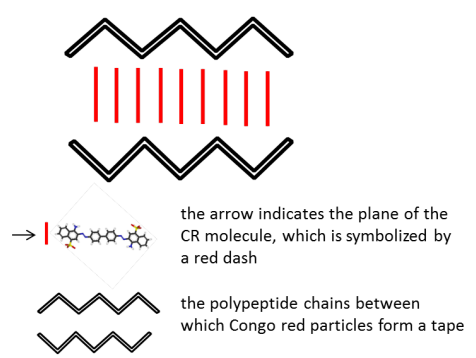

Figure 2. Congo red - a typical representative of supramolecular ribbon-like systems:

(A) an individual molecule of CR; (B) supramolecular ribbon-like structure created by individual Congo red molecules; (C) interaction of CR with polypeptide chains

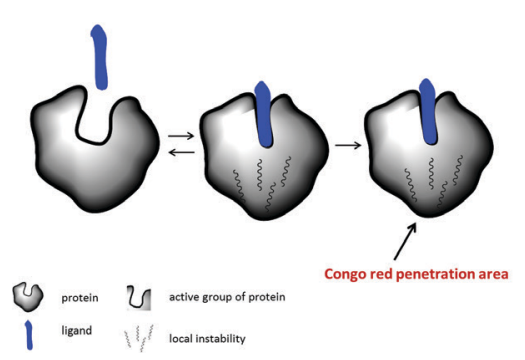

Figure 3. Local structure change in the area of the active group of the protein after ligand binding causes destabilization of the protein molecule outside the active group and, as a result, creates the possibility of binding e.g. supramolecular systems of the Congo red type

pounds of this group (Fig. 2) (Laskowski et al., 2009; McLaughlin et al., 2012).

The ribbon-like structures or ribbon fragments of Congo red type create conditions that facilitate their penetration and binding in unstable areas of protein molecules far away from the active site. Spatial changes of the active site induced by the attachment of natural ligand and by supramolecular ligand binding are coupled. If the change in protein structure enables supramolecular ligand penetration and its stable binding is dependent on the protein function, namely natural ligand binding, then reversibility of the process is suppressed. At the same time, it means that the link between the protein and naturally non-dissociable ligand is strengthened (Fig. 3). This inhibition of reversibility in the case of enzymatic processes is called uncompetitive inhibition (Fig. 4).

However, in the case of proteins which naturally form complexes (e.g. antibodies) we observe a considerable amplification of the binding. It can be observed by recording the agglutinating activity of polyclonal anti-SRBC (anti-Sheep Red Blood Cells) antibodies at increasing concentrations of Congo red. Due to the variability of the immune response, the produced pool of antibodies has polyclonal character, i.e. anti-SRBC antibodies show a differential affinity for the antigen. Only a small fraction presents the affinity sufficiently high to induce red blood cell agglutination. The remaining antibodies of the pool, which can be defined as weak, show affinities gradually decreasing to zero. In the presence of Congo red, engagement of antibodies in red blood cell agglutination rises. This engagement progresses with increasing CR concentration. This unusual phenomenon is explained - as mentioned earlier - by the elevated irreversibility of the process of $\mathrm{Ag}-\mathrm{Ab}$ complex formation. Weak antibodies remain in the complex briefly or very briefly but even a short contact with the antigen induces structural changes which enable CR complexation. It is the easier the higher CR concentration is because CR complexation is possible only in the supramolecular form, i.e. with the associated molecules while the association increases with the concentration. It is crucial that

$$
\begin{gathered}
v=\frac{V_{\max }[\mathrm{S}]}{K_{m}+[\mathrm{S}]\left(1+[\mathrm{I}] / K_{i}\right)} \\
\begin{array}{|c|l|}
\hline \mathrm{V}_{\max } & \text { maximal rate of the reaction } \\
\hline[\mathrm{S}] & \text { the concentration of the substrate } \\
\hline \mathrm{K}_{\mathrm{m}} & \text { Michaelis-Menten constant } \\
\hline[\mathrm{I}] & \text { the concentration of the inhibitor } \\
\hline \mathrm{K}_{\mathrm{i}} & \text { dissociation constant } \\
\hline
\end{array}
\end{gathered}
$$

Figure 4. Michaelis-Menten equation for uncompetitive inhibition 


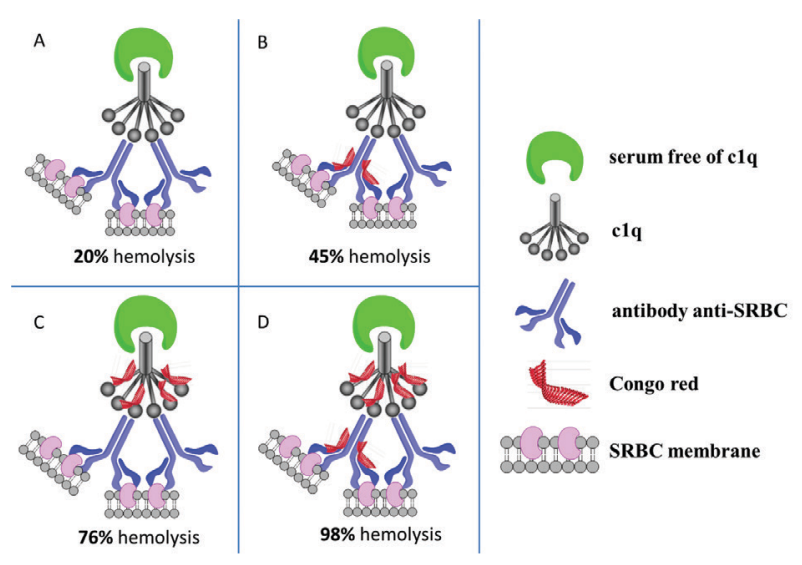

Figure 5. Effect of enhancing $c 1 q$ complexation with anti-SRBC (sheep red blood cells) antibodies when binding to SRBC with (or without) Congo red

(A) \% of hemolysis without CR; (B) \% of hemolysis with CR complexed with anti-SRBC antibodies bound to erythrocytes (Congo red is only bound to the antibodies that are stretched by binding with both arms); (C) \% of hemolysis with CR complexed with $\mathrm{C1C}$ bound to anti-SRBC antibodies interacting with erythrocytes; (D) $\%$ of hemolysis with CR complexed with $\mathrm{Clq}$ bound to anti-SRBC antibodies complexed with CR and associated with erythrocytes (Congo red is only bound to the antibodies that are stretched by binding with both arms)

the ability of the antibody to form a complex with CR is generated by the reaction of the antibody with the antigen. Free antibodies do not react with Congo red. This mechanism corresponds to uncompetitive inhibition in the case of enzymes since it perpetuates the enzyme-substrate complex existence by preventing its disintegration. In this way, it inhibits the reaction which by nature should be reversible (Piekarska et al., 2004; Roterman et al., 2018). This uniqueness of action is particularly conspicuous in the case of complement. Congo red significantly strengthens c1q complexation with antibodies but it concomitantly inhibits convertase, thus the overall effect will be the suppression of the complement action (Fig. 5) (Jagusiak et al., 2015).

Unstable proteins also react with Congo red. IgG light chains, which often pose a risk to the organism, belong to such instable proteins. For instance, these chains typically occur in the serum of myeloma patients. If the instability of these pathological serum components is significant, they can aggregate in the kidneys causing their blockade and urinary tract failure. The intensity of complexation of Congo red and its derivatives can be an early indicator of the degree of instability and thus of the risk, which can be helpful in taking the necessary preventive measures (Fig. 6) (Piekarska et al., 2001, Król et al., 2005).

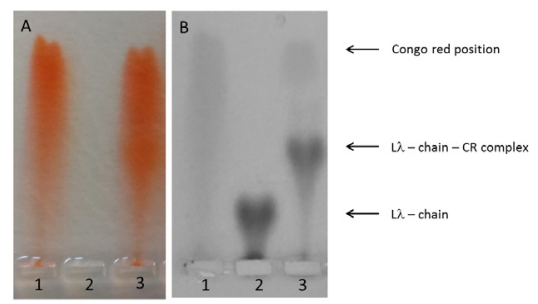

Figure 6. The light chain $(\mathrm{L} \lambda)$ complexed with Congo red migrates faster than the free protein during electrophoretic separation on an agarose gel:

(A) CR visible after electrophoretic separation; (B) samples A after visualization of the light chain (CR visible in the background); 1 , Congo red; 2 , $L \lambda$ - chain; 3 , $L \lambda$-chain and Congo red complex
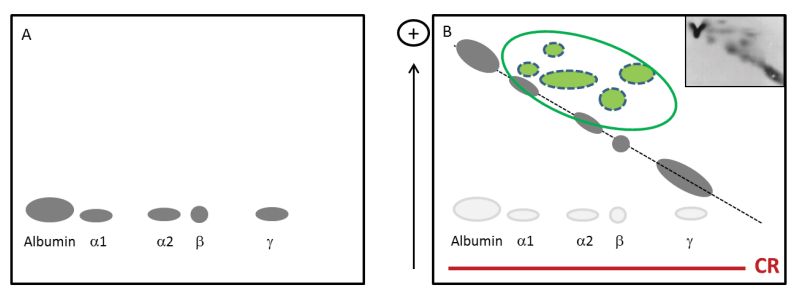

$\oplus$

Figure 7. Interaction of unstable serum proteins with Congo red: bi-directional electrophoresis images:

(A) stage I, electrophoretic separation of serum proteins (migration to the left: direction indicated by the arrow); (B) stage II, electrophoresis in the second direction (from bottom to the top: direction indicated by the arrow) of serum proteins separated in stage I, but with the addition of Congo red (red line below). Normal, non-CR-binding proteins are visible on the black dotted line. Proteins above the dotted line (marked with a green circle) are affected proteins that travel faster after binding to CR. Insert: photo of an electrophoretic separation of the serum proteins of a patient with multiple myeloma

These studies were carried out by Spólnik and others (Spólnik et al., 2003) and led to the development of the instability assessment methods. However, in the most interesting cases, protein, as a result of its function, makes available the supramolecular ligand binding sites in its structure. Such a situation can be used in clinical and diagnostic practice. Apart from antibodies, acute phase proteins can be mentioned here as examples (Wang et al., 2016). Acute phase proteins belong to the serum components. They are capable of the uptake and removal of harmful products of metabolic processes from the serum. This activity significantly rises in pathological cases, including cancer. They most often bind the proteolytic enzymes which due to specific function can penetrate to blood. They also remove cell breakdown products, which accompany especially inflammation or cancer. Free hemoglobin released as a result of red blood cell destruction may also be dangerous.

By forming complexes with substances drawn from blood, acute phase proteins become susceptible to Congo red binding. CR binding alters the protein charge making it more negative. Thus, the proteins can be separated by serum electrophoresis and provide valuable information on pathological processes progressing in the body. For that purpose, two-dimensional electrophoresis of serum proteins is carried out. During the electrophoresis in the second dimension, perpendicular to the first one, Congo red is loaded below the proteins separated in the first run. Congo red migrates fast and outruns

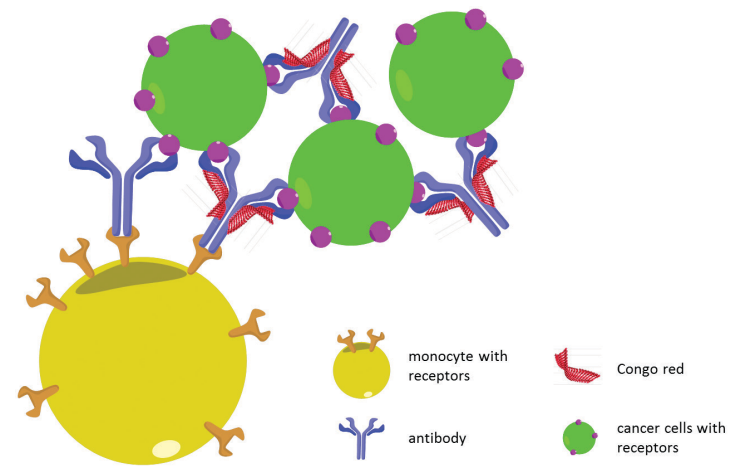

Figure 8. Model of complexing Congo red with antibodies bound by receptors present on the surface of monocytes and their interaction with cancer cells. Congo red is only bound to the antibodies that are stretched by binding with both arms. 
the migrating proteins forming complexes with those of them whose structure allows for the dye penetration.

Proteins attached to Congo red migrate faster and after electrophoresis, their bands are located above the CR-unbound proteins. The red color of Congo red is removed by reduction while proteins are fixed and visualized by classical bromophenol blue staining (Fig. 7) (Spólnik et al., 2004).

Congo red also forms complexes with albumin, apolipoproteins, alfa-1-proteinase (Roterman \& Konieczny, 1998, Stopa et al., 2006) and with receptors of interacting cells, e.g. monocytes and cancer cells (Fig. 8) (Roterman et al., 2018).

\section{RIBBON-LIKE SUPRAMOLECULAR SYSTEMS: HOST- GUEST INTERACTIONS}

Supramolecular structures reveal also other atypical properties which can find practical applications. These possibilities arise due to the non-covalent attachment of molecules in the supramolecular systems. Because of that, the foreign molecules can intercalate into the system as guest compounds, which endows the supramolecular system some new characteristics. Rhodamine B, a strongly fluorescent dye, dissolved in Congo red can be an example of such a system. Binding of such mixed system is suitable for observation of immune complex formation with the use of UV spectrometry since Rhodamine B distinctly highlights Congo red indicating that the mixed system binding to antibodies (Fig. 9) (Konieczny et al., 1997; Rybarska et al., 2004; Roterman et al., 2018).

Also, drugs can intercalate into the ribbon-like supramolecular system which can reach immune complexes capable of specific binding the supramolecular ligands. In this way, targeted drug delivery can be achieved, in this case termed immunotargeting. Such experiments were carried out using the model drug doxorubicin. The molecular modeling of doxorubicin interaction with different supramolecular systems (Congo red, Evans blue and Titan yellow) demonstrated that such mixed supramolecular systems indeed could be formed (Roterman et al., 2018; Jagusiak \& Pańczyk, 2019).

This approach can also be used for contrast application in electron microscopy. Experiments of this type were performed by binding silver ions $\left(\mathrm{Ag}^{+}\right)$with Titan yellow (TY) which besides binding the metal ions forms complexes with proteins, just like Congo red. Alternatively, both dyes (TY and CR) may be combined. In this

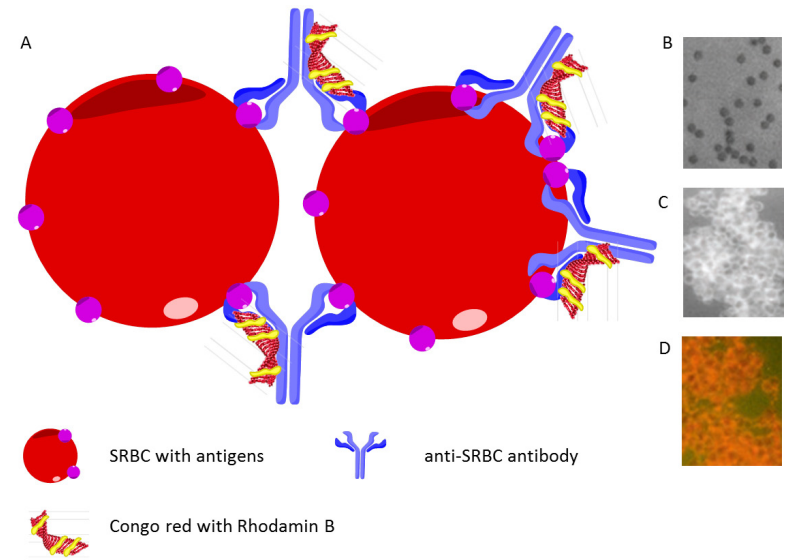

Figure 9. Model of SRBC agglutination with anti-SRBC antibodies - the effect of enhancing agglutination due to the use of CR. By complexing CR with Rhodamine $B$, the potential for immunotargeting of model compounds is increased.

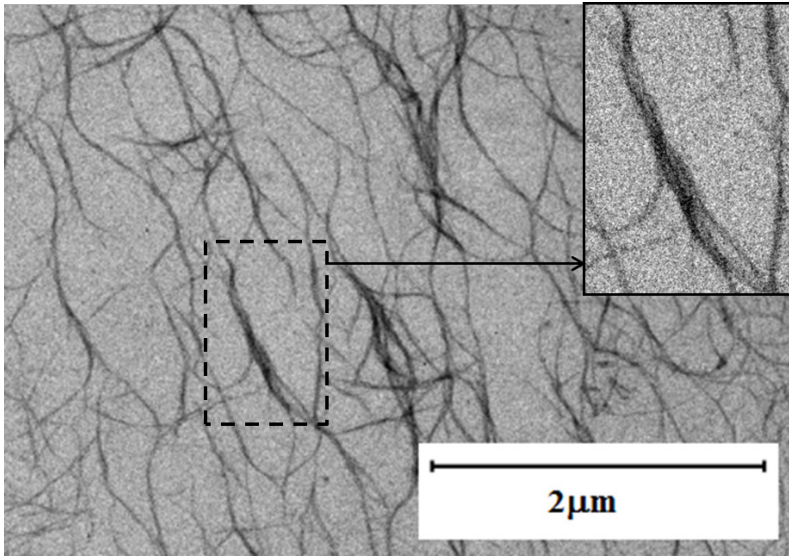

Figure 10. Amyloid fibrils beta 1-40 associated with the CR-TYAg triple complex

way, the development of amyloid fibril structures could be followed. Figure 10 presents the developing amyloid fibrils stained with the above-mentioned positive dye (Rybarska 2017).

Apart from the use of the above-described TY-Ag system in analytical techniques, its application in microbiology was tested as an alternative to antibiotic therapy. The administration of such supramolecular system has a great advantage of reducing silver toxicity. The mixed system Congo red-Titan yellow-Ag is also more soluble and is able to remove the excess of compounds from the body (Roterman et al., 2018).

\section{RIBBON-LIKE SUPRAMOLECULAR SYSTEMS: INTERACTION WITH CARBON NANOTUBES}

Carbon nanotubes provide another possibility of using supramolecular dyes as they bind large amounts of Congo red until their dissolution in aqueous solution due to acquiring a strong negative charge. It creates an opportunity to achieve a greater concentration of the dye and thus a locally higher concentration of the drug (Fig. 11). It was shown that CR interacted with carbon nanotube surface via „face to face” stacking. Despite the interaction with carbon nanotubes, CR preserves the binding capability of other molecules, e.g. model drugs (doxorubicin). Triple systems comprising nanotube-CR-doxorubicin were analyzed along with drug release and its action on cells in culture. The results revealed promising new hybrid systems created by coupling the ribbon-like supramolecular structures intercalating drugs with carbon nanotubes. These systems are characterized by a high drug binding capacity and the capability of gradual drug release at lowered $\mathrm{pH}$. Such compounds represent a promising solution in many fields of biomedicine of-
Carbon nanotube $+C R$

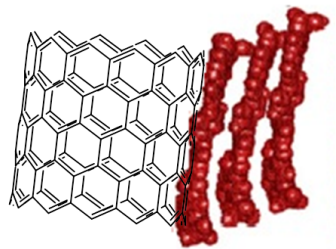

Carbon nanotube $+\mathrm{CR}+$ drug

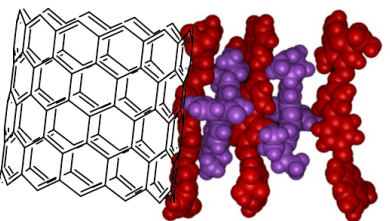

Figure 11. Carbon nanotubes provide possibility of binding Congo red supramolecular structure and mixed structures: Congo red intercalated by foreign molecules as guest compounds. 
fering a wide range of potential applications (Jagusiak et al., 2017, Jagusiak \& Pańczyk, 2019).

\section{CONCLUSIONS}

A broad array of applications of supramolecular systems results from a wide spectrum of potential advantages they provide. Different shapes of association products and the ability to form mixed structures and thus to modify the properties appear particularly attractive. Biological actions are principally the effect of the interaction of certain types of supramolecular structures with proteins by penetration of protein molecule in the area showing reduced stability. It is especially pertinent to ribbon-like systems. Instability caused by ligand binding is also encountered in the normal functioning of proteins. In such a situation, penetration of supramolecular ligand stabilizes the protein in the active state. If it affects a reversible process, like in the case of enzymes, the effect is manifested as inhibition. Conversely, if it influences a process which is sustainable by nature, then it results in its enhancement. Understandably, supramolecular ligand binding to proteins is attractive especially if mixed structures are created because a drug can become the guest compound intercalating the supramolecular system. Since the ribbon-like supramolecular structures eagerly bind to the immune complexes, it creates an opportunity for the development of immunotargeting techniques.

Supramolecular ligands can also be used in diagnostic procedures because they are able to change physicochemical properties of proteins upon the formation of mixed structures, enabling the system to interact with selected objects.

Congo red, commonly accepted as a standard system, is virtually non-toxic since it does not cross plasma membranes of normal cells and is quickly excreted from the organism. However, it can be toxic when ingested because $\mathrm{CR}$ is reduced by intestinal bacteria to form benzidine which is thought to be carcinogenic. However, this danger can be reduced by the consumption of high-cellulose products during CR administration since cellulose strongly binds $\mathrm{CR}$ and radically diminishes its susceptibility to reduction.

In comparison to the known supramolecular systems presented to date, the ribbon-like structures seem to be particularly interesting because they can be applied both in diagnostics and development of precise drug delivery systems and as an alternative to increasingly ineffective antibiotic therapy. Based on the studies conducted so far, it can be expected that investigations of these interesting systems will bring new efficient solutions both in therapy and diagnostics in the future.

\section{REFERENCES}

Atwood JL, Steed JW (2004) Encyclopedia of Supramolecular Chemistry. CRC Press Tylor\&Francis Group. https://doi. org/10.1201/9780429075728

Chen P, Mondal JH and Zhou Y, Zhua H, Shi B (2016) Construction of a neutral linear supramolecular polymer via orthogonal donor-acceptor interactions and pillar 5 arene-based molecular recognition. Polym Chem 7: 5221-5225. https://doi.org/10.1039/C6PY01123C

Jagusiak A, Konieczny L, Krol M, Marszalek P, Piekarska B, Piwowar P, Roterman I, Rybarska J, Stopa B, Zemanek G (2015) Intramolecular immunological signal - hypothesis revieved - structural background of signalling revealed by using Congo red as a specific tool. Mini Rev Med Chem 14: 11. https://doi.org/10.2174/1389557514666141127150803

Jagusiak A, Piekarska B, Pańczyk T, Jemioła-Rzemińska M, Bielańska E, Stopa B, Zemanek G, Rybarska J, Roterman I, Konieczny L (2017) Dispersion of single-wall carbon nanotubes with supramolecular Congo red - properties of the complexes and mechanism of the interaction Beilstein J Nanotechnol 8: 636-648. https://doi.org/10.3762/bjnano.8.68
Jagusiak A, Pańczyk T (2019) Interaction of Congo Red, Evans Blue and Titan Yellow with doxorubicin in aqueous solutions. A molecular dynamics study. J Mol Liquids 279: 640-648. https://doi.org/10.1016/j. molliq.2019.02.012

Jagusiak A, Chłopaś K, Zemanek G, Jemioła-Rzemińska M, Piekarska B, Stopa B, Pańczyk T (2019) Self-assembled supramolecular ribbon-like structures complexed to singlewalled carbon nanotubes as possible anticancer drug delivery systems. Int J Mol Sci 20: 2064. https://doi. org/10.3390/ijms20092064

Kang JC, Kumar V, Yang D, Chowdhurya PR, Hohlb RJ (2002) Cyclodextrin complexation: influence on the solubility, stability, and cytotoxicity of camptothecin, an antineoplastic agent. Eur I Pharm Sci 15: 163-170. https://doi.org/10.1016/S0928-0987(01)00214-7

Konieczny L, Piekarska B, Rybarska J, Skowronek M, Stopa B, Tabor B, Dabroś W, Pawlicki R, Roterman I (1997) The use of congo red as a lyotropic liquid crystal to carry stains in a model immunotargeting system-microscopic studies. Folia Histochem Cytobiol 35: 203-210

Król M, Roterman I, Piekarska B, Konieczny L, Rybarska J, Stopa B, Spólnik P, Szneler E (2005) An approach to understand the complexation of supramolecular dye Congo red with immunoglobulin $\mathrm{L}$ chain lambda. Biopolymers 77: 155-162. https://doi.org/10.1002/bip.20197

Laskowski RA, Gerick F, Thornton JM (2009) The structural basis of allosteric regulation in proteins. FEBS Lett 583: 1692-1698. https://doi. org/10.1016/j.febslet.2009.03.019

McLaughlin RN Jr, Poelwijk FJ, Raman A, Gosal WS, Ranganathan R (2012) The spatial architecture of protein function and adaptation. $\mathrm{Na}$ ture 491: 138-142. https://doi.org/10.1038/nature11500

Piekarska B, Konieczny L, Rybarska J, Stopa B, Zemanek G, Szneler E, Król M, Nowak M, Roterman I (2001) Heat-induced formation of a specific binding site for self-assembled Congo red in the $\mathrm{V}$ domain of immunoglobulin L chain lambda. Biopolymers 59: 446-456. https://doi. org/10.1002/1097-0282(200111)59:6<446::AID-BIP1049>3.0.CO;2-X

Piekarska B, Konieczny L, Rybarska J, Stopa B, Spólnik P, Roterman I, Król M (2004) Intramolecular signaling in immunoglobulins - new evidence emerging from the use of supramolecular proteins ligands $J$ Physiol Pharmacol 55: 487-501. PMID: 15381822

Roterman I, Konieczny L (2018) Self-assembled molecules - new kind of protein ligands. Supramolecular ligands. Springer Open https://doi. org/10.1007/978-3-319-65639-7

Roterman I, Rybarska J, Konieczny L, Skowronek M, Stopa B, Piekarska B, Bakalarski G (1998) Congo red bound to $\alpha$-1-proteinase inhibitor as a model of supramolecular ligand and protein complex. Comput Chem 22: 61-70. https://doi.org/10.1016/S0097-8485(97)00014-4

Rybarska J, Piekarska B, Stopa B, Spólnik P, Zemanek G, Konieczny L, Roterman I (2004) In vivo accumulation of self-assembling dye Congo red in an area marked by specific immune complexes: possible relevance to chemotherapy. Folia Histochem Cytobiol 42: 101-110.

Rybarska J, Konieczny L, Jagusiak A, Chłopaś K, Zemanek G, Piekarska B, Stopa B, Piwowar P, Woźnicka O, Roterman I (2017) Silver ions as EM marker of congo red ligation sites in amyloids and amyloid-like aggregates, Acta Biochim Pol 64: 161-169 https://doi.org/10.18388/ abp.2016_1393

Schneider HJ (2013) Supramolecular Systems in Biomedical Fields. RSC Publishing. ISBN 978-1-84973-658-9

Spólnik P, Piekarska B, Stopa B, Konieczny L, Zemanek G, Rybarska J, Król M, Nowak M, Roterman I (2003) The structural abnormality of myeloma immunoglobulins tested by Congo red binding. Med Sci Monit 9: 145-153. PMID: 12709666

Spólnik P, Konieczny L, Piekarska B, Rybarska J, Stopa B, Zemanek G, Król M, Roterman I (2004) Instability of monoclonal myeloma protein may be identified as susceptibility to penetration and binding by newly synthesized Congo red derivatives. Biochimie 86: 397-401. https://doi. org/10.1016/j.biochi.2004.05.002

Stopa B, Rybarska J, Drozd A, Konieczny L, Król M, Lisowski M, Piekarska B, Roterman I, Spólnik P, Zemanek G (2006) Albumin binds self-assembling dyes as specific polymolecular ligands. Int J Biol Macromol 40: 1-8. https://doi.org/10.1016/j.ijbiomac.2006.05.002

Szejtli J (1988) Cyclodextrin technology. Springer-Science + Business Media. https:/ / doi.org/10.1007/978-94-015-7797-7

Wang Y, Liu Y, Deng X, Cong Y, Jiang X (2016) Peptidic $\beta$-sheet binding with Congo Red allows both reduction of error variance and signal amplification for immunoassays. Biosens Bioelectron 86: 211-218. https://doi. org/10.1016/j.bios.2016.06.021

Webber MJ, Appel EA, Meijer EW, Langer R (2016) Supramolecular biomaterials. Nat Mater 15: 13-26. doi.org/10.1038/nmat4474

Wells JA, McClendon CL, Zhua H, Shi B (2007) Reaching for high-hanging fruit in drug discovery at protein-protein interfaces Nature 450: 10011009. https:// doi.org/10.1038/nature06526

Xin J, Lijuan Z, Bai X, Xinyuan Z, Deyue Y (2019) Chemistry and Molecular Medicine Supramolecular nanoscale drug-delivery system with ordered structure. Nat Sci Rev 6: 1-10. https://doi.org/10.1093/nsr/nwz018

Yu ZL, Erbas A, Tantakitti F, Palmer LC, Jackman JA, Olvera de la Cruz M, Cho NJ, Stupp SI (2017) Co-assembly of peptide amphiphiles and lipids into supramolecular nanostructures driven by anion-pi interactions. JAm Chem Soc 139: 7823-7830. https://doi.org/10.1021/jacs.7b02058 